\title{
The Aspergillus nidulans fluG gene is required for production of an extracellular developmental signal and is related to prokaryotic glutamine synthetase I
}

\author{
Bee Na Lee and Thomas H. Adams \\ Department of Biology, Texas A \& M University, College Station, Texas 77843 USA
}

\begin{abstract}
Mutations in the Aspergillus nidulans fluG gene disrupt the programmed induction of asexual sporulation and result in formation of fluffy colonies that are characterized by undifferentiated cotton-like masses of vegetative cells. We show that the fluG mutant phenotype is suppressed when fluG mutant colonies are grown next to wild-type colonies even if the two strains are separated by dialysis membrane with a 6000- to 8000-dalton pore size. flu $G$ encodes a cytoplasmically localized $\sim 96,000$-dalton polypeptide that is present at relatively constant levels during vegetative growth and following developmental induction. Sequence analysis of fluG demonstrated that the carboxy-terminal 436 amino acids predicted by the 864-codon FluG open reading frame shares $\sim 28 \%$ identity with GSI-type prokaryotic glutamine synthetases. We consider it unlikely that FluG functions in synthesis of glutamine but instead propose that FluG functions as a GSI-related enzyme in synthesizing an extracellular signal directing asexual sporulation and perhaps other aspects of colony growth. The relationships between flu $G$ and other genes identified by fluffy mutants are discussed.
\end{abstract}

[Key Words: Differentiation; asexual sporulation; extracellular signaling; filamentous fungi]

Received December 3, 1993; revised version accepted January 27, 1994.

Aspergillus nidulans is an ascomycetous filamentous fungus that grows by forming an ordered network of hyphal cells, called a mycelium, resulting in a radially symmetric colony that expands at a constant rate. At a set time after spore germination, some of the hyphal cells within the center of the mycelium produce aerial branches that will develop into complex, multicellular, spore-bearing structures termed conidiophores (Axelrod 1972; B. Lee and T., Adams in prep.|. Following onset of conidiation there is a rapid spread toward the outer edge, so that in mature colonies, the youngest conidiophores are observed $1-2 \mathrm{~mm}$ behind the growing edge, and the oldest conidiophores are found in the center. The molecular signals coordinating colony morphology and regulating initiation of conidiophore development are not known. However, these processes are apparently programmed into the life cycle of $A$. nidulans rather than occurring strictly as a response to unfavorable environmental circumstances as is observed in many other microorganisms.

Developmental induction ultimately results in activation of an early transcriptional regulator for conidiationspecific genes, called brlA (Clutterbuck 1969; Boylan et al. 1987). Activation of $b r l A$ expression likely represents a major control point in the conidiation pathway because forced expression of brlA at any time during the life cy- cle is sufficient to activate development of simple conidiophores that produce viable spores (Adams et al. 1988). We showed previously that fluG (formerly called $a c o D$ ), $f l b A$, and several other genes are required for the programmed switch from mycelial growth to activation of brlA expression and conidiophore development (Adams et al. 1992; B. Lee and T. Adams, in prep.). Mutations in all of these genes result in fluffy colonies that proliferate as undifferentiated masses of vegetative mycelia. However, when flu $G^{-}$mutants are grown under nutrient limiting conditions, this requirement is partially overcome, and some limited conidiation results (Adams et al. 1992). This observation led us to suggest that brlA can be activated in more than one way. The major sporulation pathway observed in wild-type strains requires flu $G$ and involves nutrient-independent programmed initiation of development. In flu $G^{-}$mutant strains, brlA expression and development are regulated through a mechanism that involves sensing changes in growth rate or nutrient status.

The mechanisms through which $f 1 b A, f l u G$, and other fluffy genes cause $b r l A$ activation and conidiation are not known. The importance of $f l b A$ in controlling $b r l A$ expression is made clear by the fact that overexpression of $f l b A$ in growing hyphae causes $b r l A$ activation and initiates the sporulation pathway (B. Lee and T. Adams, 
in prep.). Because $f l b A$ is related to the Saccharomyces cerevisiae SST2 gene, which is involved in regulating the signaling pathway for responding to mating pheromone, we proposed that FlbA plays a role in an intracellular signaling pathway to control Aspergillus conidiation (Dietzel and Kurjan 1987; Kurjan 1992; B. Lee and T. Adams, in prep.). In this paper we demonstrate that the conidiation defect observed in flu $G^{-}$deletion mutants can be rescued by growth next to wild-type strains, suggesting that fluG is required for producing an extracellular conidiation signal. fluG encodes an $\sim 96-\mathrm{kD}$ polypeptide that is present early in vegetative growth as well as in development and is primarily localized in the cytoplasm. The carboxy-terminal half of the FluG protein has significant similarity to the GSI class of glutamine synthetases that have previously been observed only in prokaryotes. We propose that FluG functions as an enzyme for production of an extracellular signal that controls initiation of sporulation.

\section{Results}

Extracellular rescue of the fluG mutant phenotype

When fluG mutants are grown on complete medium they produce a large mass of undifferentiated aerial mycelia giving the colony a fluffy appearance (Adams et al. 1992). Figure 1 shows that when a fluG deletion mutant that also carried a spore-color mutation resulting in yellow-spored conidiophores $(y A 2)$ was grown on complete medium next to a green-spored wild-type strain, yellow-spored conidiophores were produced at the interface between the two colonies (Fig. 1A). Yellow spores recovered from this region were plated on complete media and gave rise to fluffy mutant colonies indicating that they remained fluG $G^{-}$(not shown). fluG deletion mutants also sporulated as the colony margin approached the margin of several developmental mutants including brlA (Fig. 1B), flbA (Fig. 1C), and all other fluffy mutant strains that we have isolated to date (not shown). However, no sporulation was observed as two fluG mutant colonies merged, indicating that sporulation did not result from growth inhibition as colonies grew together (Fig. 1D).

The observed phenotypic complementation of the flu $G^{-}$conidiation defect might result from an intercellular passage of materials following hyphal anastomosis or from extracellular exchange of a conidiation signal. To test the hypothesis that cellular contact with wild type was required for phenotypic complementation of flu $G$ mutants, a wild-type strain was inoculated on complete media and then covered with dialysis membrane having a 6000- to 8000-dalton cutoff. Under these conditions, the wild-type strain could not produce aerial structures and thus grew only as vegetative hyphae. A fluG ${ }^{-}$deletion mutant was inoculated on top of the dialysis membrane and allowed to grow until the colony margin overtook the margin of the wild-type strain. As shown in Figure 1F, yellow-spored conidiophores were produced by the flu $G^{-}$strain as it grew over the wild-type strain. As described above, when yellow spores were recovered from this region and plated on complete medium, they produced fluffy colonies, indicating that they remained flu $G^{-}$. This result demonstrates that conidiophore development by the wild-type strain is not required for rescue of the fluffy phenotype and supports the notion that complementation occurred extracellularly. However, the observed conidiation could also be explained if either the wild-type or mutant hyphae were able to penetrate the membrane, resulting in cell-cell contact. This possibility is highly unlikely because membrane recovered after the experiment remained impermeable to both Blue dextran $(\sim 2,000,000$ daltons $)$ and bovine serum albumin $(66,000$ daltons), each of which is significantly smaller than Aspergillus hyphae ( $\sim 3 \mu \mathrm{m}$ diam.). In addition, when either fluG mutants or wild-type strains were grown beneath dialysis membrane, they were never observed growing through to the surface even as long as 3 days after conidiation was first observed. Finally, no conidiation was seen for a fluG mutant strain grown on top of the membrane when a fluG mutant strain, rather than wild type, was grown beneath the membrane (not shown).

\section{fluG is related to prokaryotic glutamine synthetase}

The DNA sequence of the fluG genomic region (Adams et al. 1992) and a nearly full-length fluG cDNA clone (including the entire coding region) was determined and is presented in Figure 2. The cDNA sequence contains a single long open reading frame that is predicted to encode a 864-amino-acid polypeptide with a molecular mass of 96,389 daltons. We compared the predicted FluG sequence with other sequences in the various data bases by use of the BLAST search algorithm (Altschul et al. 1990) and found that the carboxy-terminal 436 amino acids were related primarily to a number of GSI-type glutamine synthetases seen only in prokaryotes. Limited similarity between FluG and GSII-type glutamine synthetases found in all eukaryotes and some prokaryotes was observed but only in regions shared between GSI and GSII. The most significant matches observed were to glutamine synthetase from the Gram-positive eubacterium Bacillus cereus ( $28 \%$ identity, $50 \%$ similarity; Fig. 3 and from the archaebacterium Methanococcus voltae $127 \%$ identity, $49 \%$ similarity, Nakano et al. 1989; Possot et al. 1989|. Figure 3 shows a BESTFIT comparison between the 436 amino acids at the carboxyl terminus of FluG and $B$. cereus $\mathrm{Gln} A$. The most highly conserved regions (boxed) correspond to the parts of glutamine synthetases that have been proposed to comprise the active site for the enzyme, and some of the shared amino acids in these regions are also shared with eukaryotic glutamine synthetases (Almassy et al. 1986; Janssen et al. 1988). Although some of the shared amino acids observed between FluG and GlnA are found in these conserved glutamine synthetase domains, there are several other highly conserved regions. Sequence analysis of one temperature-sensitive fluG allele, fluG684, showed the 
A B

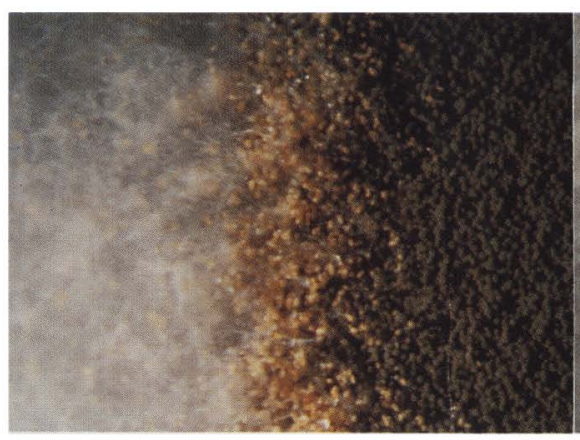

$\triangle$ fluG
Wild type

D

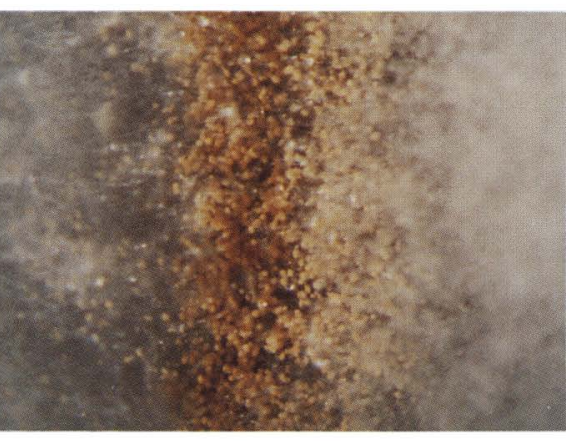

$\Delta f l u G$ $\triangle b r l A$

C

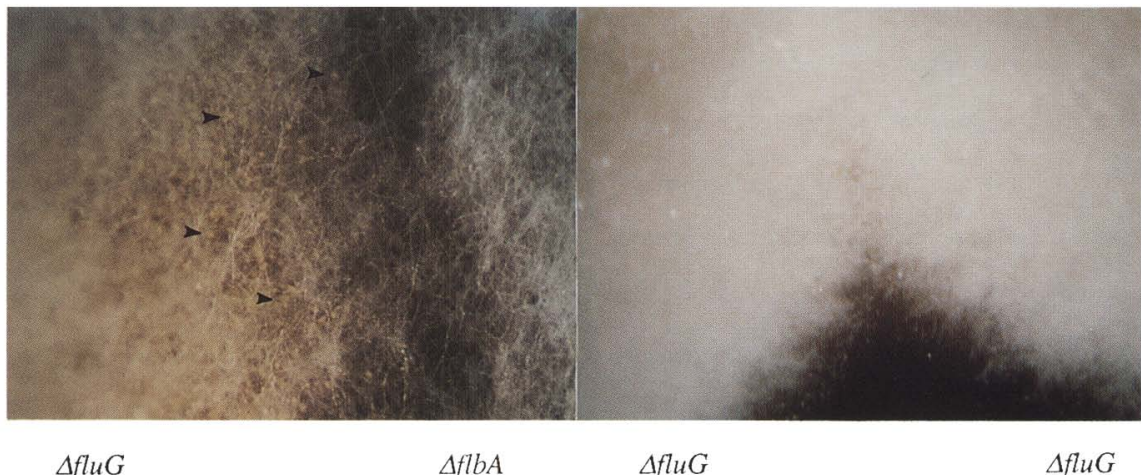

E

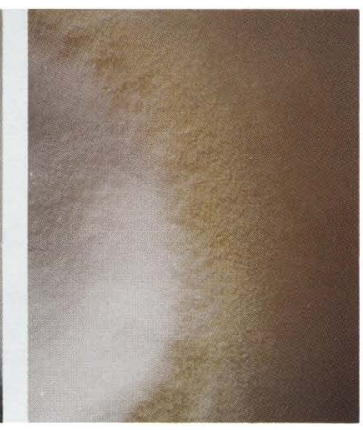

$\Delta f l u G$

$\triangle f l b A$

$\Delta f l u G$

$\Delta f l u G$

Figure 1. fluG mutants conidiate when grown adjacent to wild type strains. A yellow-spored fluG deletion mutant (TTA127.2) was

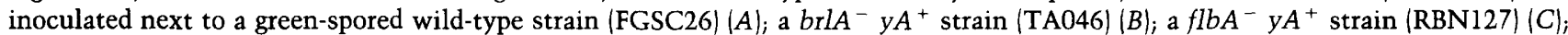
or a second fluG deletion mutant strain (TTA127.4) $(D)$ and allowed to grow on complete medium. Yellow-spored conidiophores formed at the interface between two colonies in $A-C$ (arrowheads indicate conidiophores in $C$ ) were produced by the flu $G^{-}$strain but no conidiation was observed at the interface between the two flu $G^{-}$colonies shown in $D$. (E) A wild-type strain (FGSC26) was inoculated on complete medium under a dialysis membrane with a 6000 - to 8000-dalton cutoff and the yellow-spored fluG deletion mutant (TTA127.4) was inoculated above the membrane. Yellow-spored conidiophores were produced by the fluG deletion mutant as it grew over the wild-type colony.

defect resulted from a single base-pair change converting tyrosine 773 in the glutamine synthetase-like domain to an asparagine (Figs. 2 and 3). A high degree of identity $(64 \%)$ was also observed between the last 75 amino acids in FluG and a potential open reading frame upstream of the Aspergillus niger argB gene, suggesting that a fluG homolog is linked closely to $\arg B$ in $A$. niger. No signif- icant similarity was observed between sequences in the amino terminus of FluG and other proteins in the data bases.

Although fluG is related to glutamine synthetase, another gene $(g \ln A)$ has been identified in $A$. nidulans that likely encodes the structural gene for glutamine synthetase (MacDonald 1982; Cornwell and MacDonald 1984). 


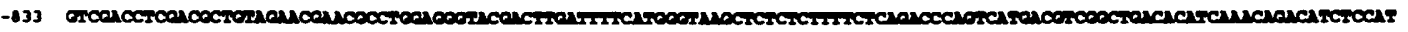

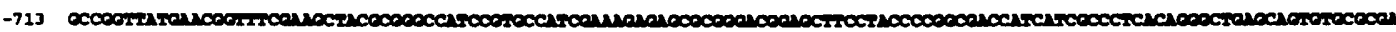

-593 cenuтcom

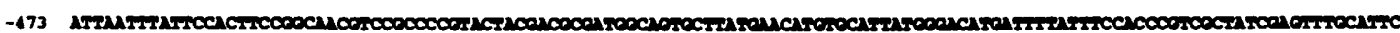

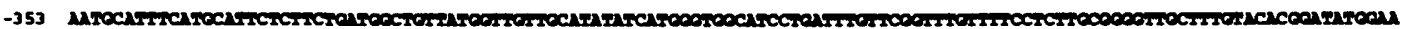

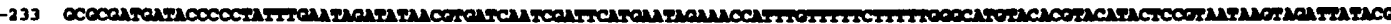

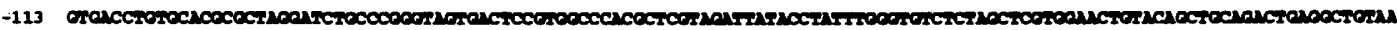

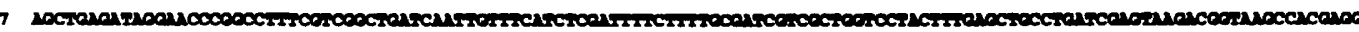

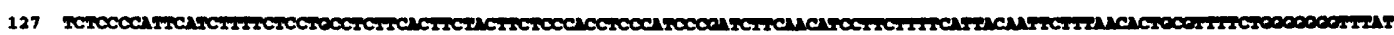

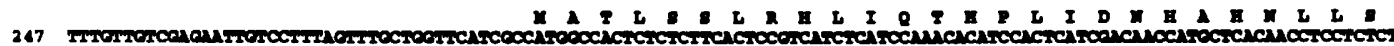

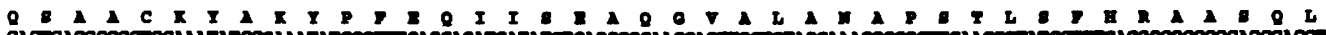

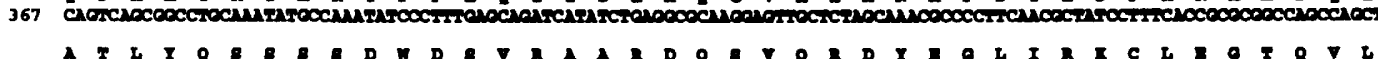

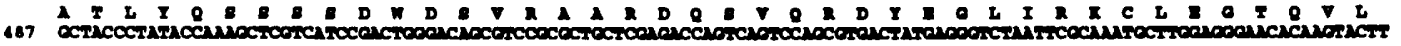

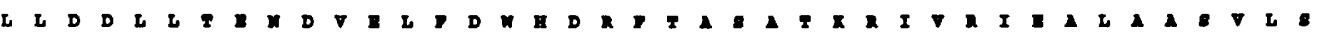

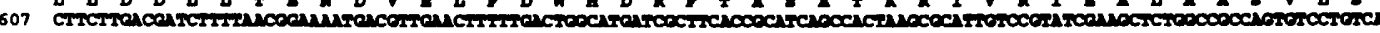

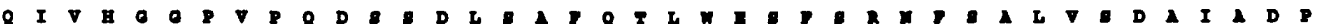

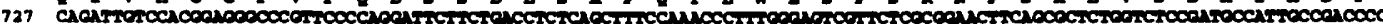

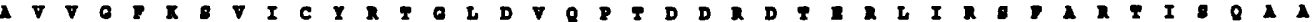

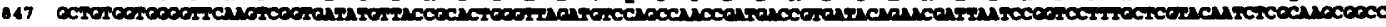

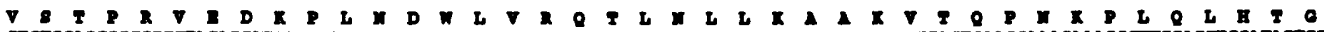

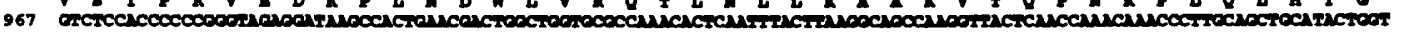

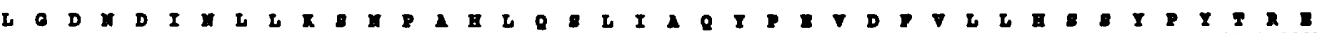
1087 Trecoanat

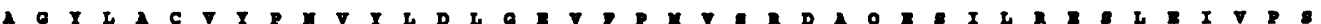

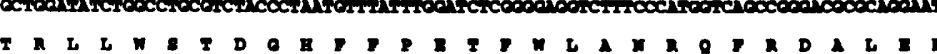

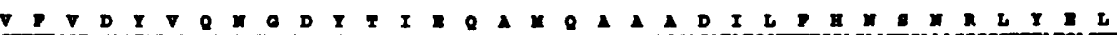

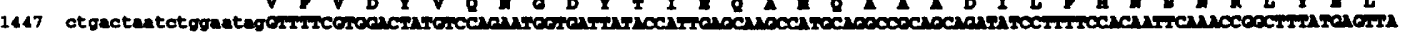

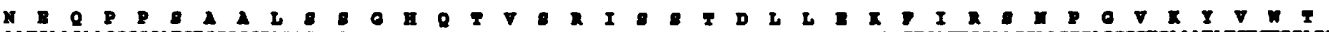

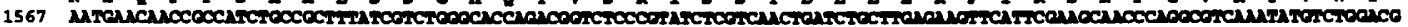

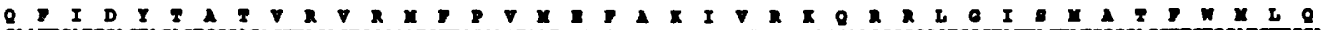

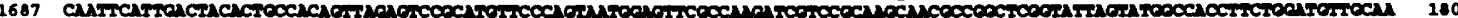

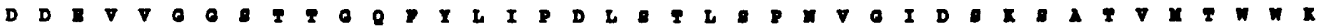

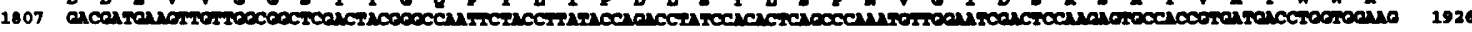

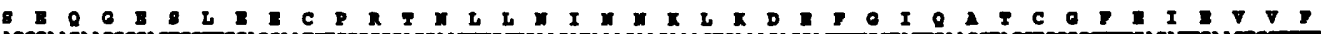
1927 iscenucubocen

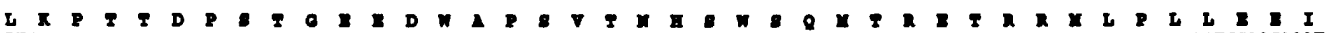

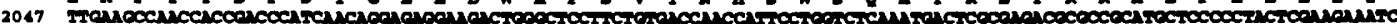

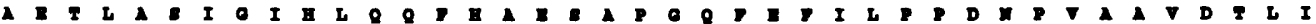

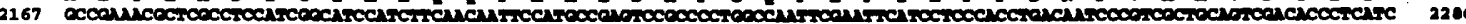

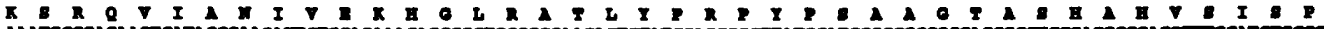

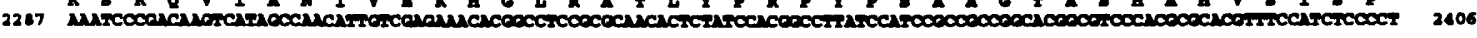

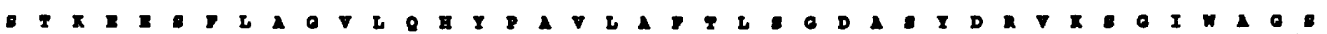

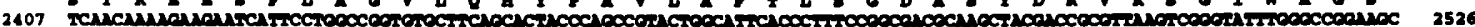
I V I

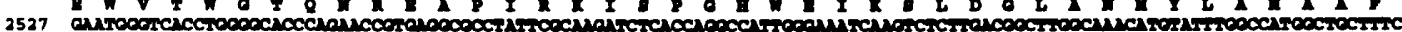

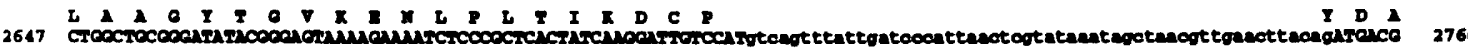

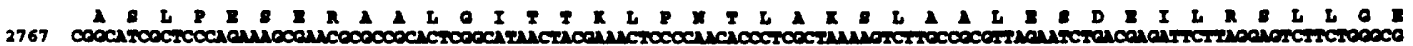

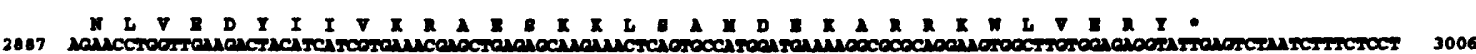

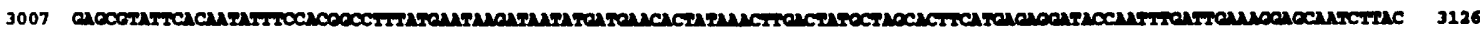

Figure 2. Sequence of the fluG-coding region. The DNA sequence of the fluG gene region and predicted amino acid sequence of the FluG polypeptide are shown. A major transcription initiation site was identified by primer extension and is designated +1 . Introns are shown in lowercase, and intron/exon junctions were determined by comparing genomic sequence with cDNA sequence. The GenBank accession number for this sequence is $\mathrm{L} 27817$.

However, all of the mutations isolated in $g \ln A$ are somewhat leaky and grow slowly in the absence of glutamine. We tested the possibility that the leaky growth phenotype of $g \ln A^{-}$mutants was attributable to an auxiliary glutamine synthetase activity derived from FluG by constructing a flu $G^{-}, g \ln A^{-}$double mutant strain. The double mutant strain grew as well as the $g \ln A^{-}$single mutant without added glutamine, indicating that flu $G$ is not required for the limited growth observed under these conditions (not shown).

\section{fluG is required for repression of fluG expression}

In earlier experiments, we constructed a fluG mutant strain by inserting the entire $\arg B$ gene region into a restriction site in the center of the fluG-coding se- 


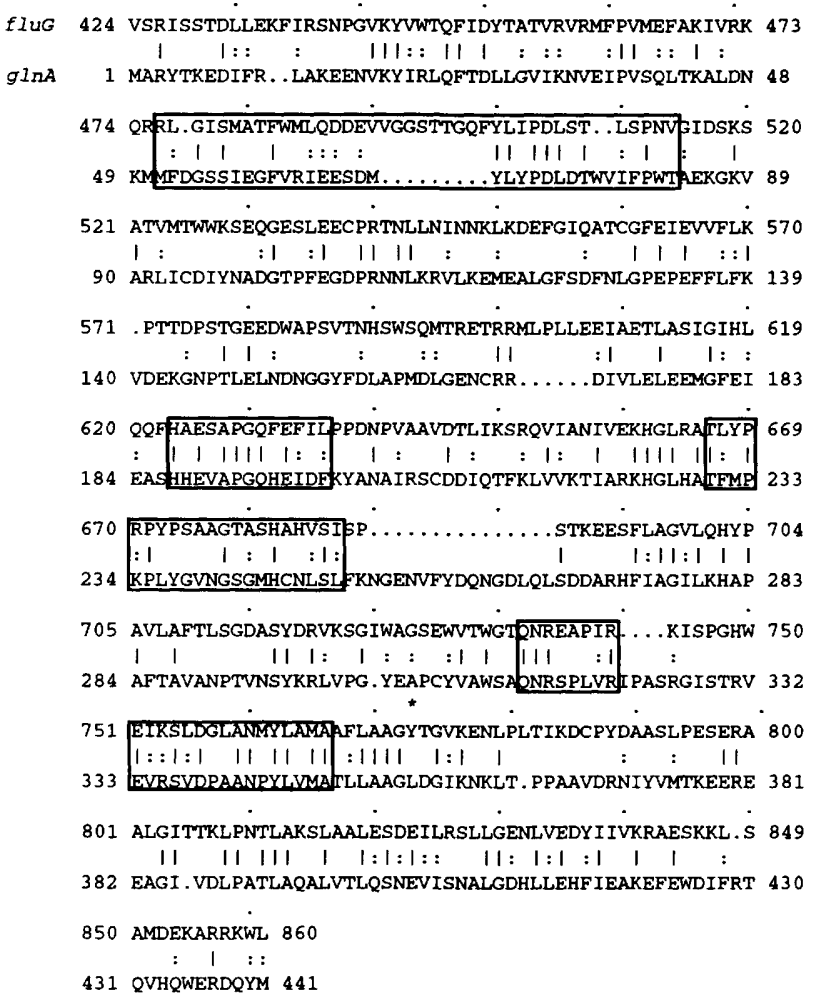

Figure 3. FluG is related to prokaryotic glutamine synthetase. A BESTFIT comparison between the carboxy-terminal half of FluG and the $B$. cereus GlnA-encoded polypeptide is shown (Nakano et al. 1989). Identities are illustrated by vertical dashes, and conservative changes are illustrated by dots. The boxed regions correspond to domains that are partially conserved between GSI and GSII and represent components of the active site. The asterisk $\left({ }^{*}\right)$ indicates tyrosine 773 , which was converted to an asparagine by the fluG684 mutation.

quences (Adams et al. 1992). Figure 4A shows that when RNA was isolated from this strain and probed with fluG DNA sequences, the flu $G$ mRNA had not only increased in size but also in abundance. This result could be caused by the insertion of $\arg B$ altering mRNA stability or disrupting transcriptional regulatory sequences. Alternatively, the observed increase in fluG transcript levels could result from the fluG mutation, indicating that fluG is in some way required to negatively regulate its own expression. To distinguish between these two possibilities, we constructed a translational gene fusion between fluG-coding sequences and the Escherichia coli gene $l a c Z$ so that flu $G$ expression could be monitored as $\beta$-galactosidase activity. Figure $4 \mathrm{~B}$ shows that fluG-directed $\beta$-galactosidase activity was four to five times higher in a fluG deletion mutant than in a wild-type strain, supporting the notion that flu $G$ negatively regulates its own expression.

\section{FluG protein is localized in the cytoplasm}

The time of appearance of FluG was examined by immunoblot analysis of protein extracts from vegetative and developing $A$. nidulans cultures with antisera directed against FluG protein produced in E. coli (Materials and methods). Figure 5A shows that the FluG antisera recognized two polypeptides that were present in a wildtype strain and in a flbA deletion mutant but were absent in a fluG deletion mutant. As was observed for fluG mRNA (Adams et al. 1992), the major $\sim 96-\mathrm{kD}$ protein was present at relatively constant levels in both young and old hyphal cultures as well as after developmental

A $\begin{array}{llllllllll}1 & 2 & 3 & 4 & 5 & 6 & 7 & 8 & 9 & 101112\end{array}$

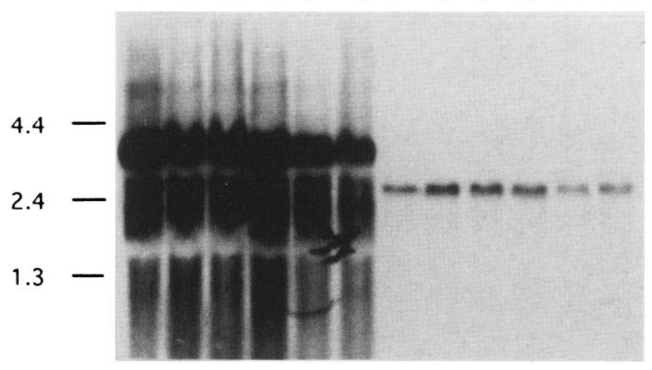

B

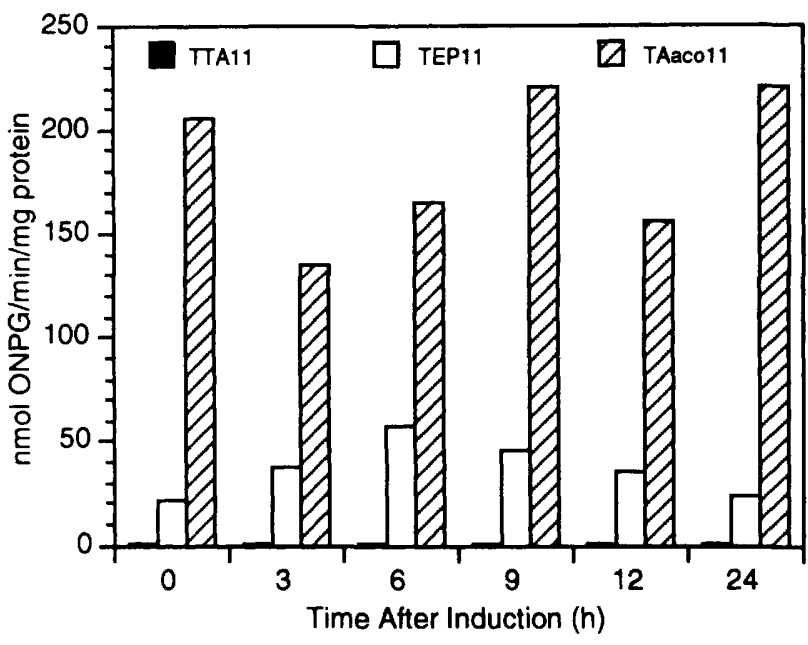

Figure 4. fluG expression is negatively autoregulated. (A) Total RNA was isolated from vegetative and developing cultures of a wild-type $A$. nidulans strain (FGSC26, lanes 7-12) and from a fluG mutant strain (TA125, lanes 1-6) containing an $\arg B$ insertion in the center of fluG and fractionated on a formaldehyde-agarose gel. The resultant gel blot was probed with an internal $2.5 \mathrm{-kb} X$ hoI fragment from fluG. (Lanes 1,7 ) $20 \mathrm{hr}$ of vegetative growth; (lanes 2,8$) 3 \mathrm{hr}$ after developmental induction; (lanes 3,9) $6 \mathrm{hr}$ after developmental induction; (lanes 4,10) $9 \mathrm{hr}$ after developmental induction; (lanes 5,11 ) $12 \mathrm{hr}$ after developental induction; (lanes 6,12) $24 \mathrm{hr}$ after developmental induction. (B) fluG ${ }^{+}$(TEP11) and fluG ${ }^{-}$(TAacol1) strains containing a translational fluG::lacZ fusion integrated at the $\operatorname{trp} C$ locus were grown for $20 \mathrm{hr}$ in liquid culture (time 0 ) and then induced to develop by exposing to an air interface. Samples were taken at the times indicated, and cell extracts were assayed to determine $\beta$-galactosidase levels. The control strain (TTAll) contains plasmid sequences integrated at $\operatorname{trp} C$ but lacks fluG and $l a c Z$ sequences. 
A

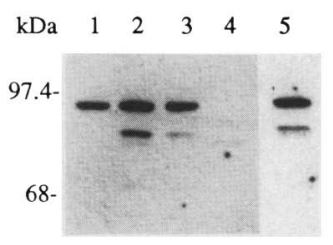

B

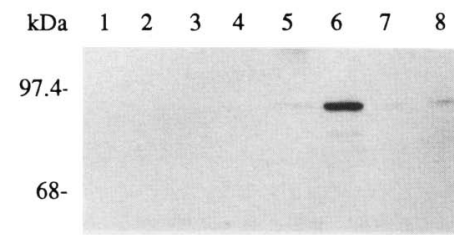

Figure 5. FluG is a cytoplasmic protein present in vegetative and developing cultures. (A) Total protein was extracted from $A$. nidulans strains FGSC26 (wild type), TTA127.4 ( $\Delta$ fluG), and rBN127 $(\triangle f l b A)$ grown under various culture conditions, and electrophoretically separated, and FluG protein was identified using a FluG-specific polyclonal antibody. (Lanes 1-3) $50 \mu \mathrm{g}$ of total protein from FGSC26 grown in liquid culture for $12 \mathrm{hr}$ (lane 1) or $20 \mathrm{hr}$ (lane 2), and from the same strain $8 \mathrm{hr}$ after developmental induction (lane 3); (lane 4) $50 \mu \mathrm{g}$ of total protein from TTA127.4 grown in liquid culture $20 \mathrm{hr}$; (lane 5) $50 \mu \mathrm{g}$ of total protein from rBN127 grown in liquid culture $20 \mathrm{hr}$. $(B)$ Total protein was isolated from subcellular fractions from strains TTA127.4 (Lanes 1-4) or FGSC26 (lanes 5-8) that had been grown for $20 \mathrm{hr}$ in liquid culture, and separated electrophoretically, and FluG protein was detected by use of the FluG antibody. (Lanes 1,5) $50 \mu \mathrm{g}$ from the nuclear protein fraction; (lanes 2,6) $50 \mu \mathrm{g}$ from the soluble protein fraction; (lanes 3,7) 50 $\mu \mathrm{g}$ from the mitochondrial protein fraction; (lanes 4,8$) 50 \mu \mathrm{g}$ of protein from the membrane fraction.

induction. The $\sim 75-\mathrm{kD}$ polypeptide was observed primarily in the 20-hr hyphal culture but was also detectable after developmental induction. The bands corresponding to FluG were not observed in immunoblots prepared with preimmune serum as the primary antibody (not shown).

The subcellular location of FluG was examined by immunoblot analysis of fractionated cell extracts (Materials and methods) with the antisera directed against FluG protein. Figure 5B shows that the majority of the 96- and 75-kD FluG polypeptide was cytoplasmically localized in the wild-type strain. Neither polypeptide was detected in extracts from the fluG deletion mutant.

\section{Genetic interaction between flbA and fluG}

One other early developmental gene, $f l b A$, has been shown to be required for the expression of $b r l A$ under normal growth conditions, and overexpression of $f l b A$ in vegetative hyphae resulted in activation of brlA expression and development (B. Lee and T. Adams, in prep.). We addressed the possibility that $f l u G$ and $f l b A$ form a linear pathway regulating the activation of $b r l A$ expression during $A$. nidulans conidiophore development by examining their epistatic relationship. A meiotic cross between flbA and fluG deletion mutant strains (TBN39.5 and TTA127.2) resulted in 25\% fluG-like, $50 \% \mathrm{flbA}$-like, and $25 \%$ wild-type progeny as would be expected if the $f l b A^{-}$flu $G^{-}$double mutant had a $f l b A^{-}$ phenotype. This interpretation was confirmed with Southern blot analysis to demonstrate the presence of $f l b A$ and $f l u G$ deletions in the progeny (data not shown).

Close examination of one double mutant strain (RBN130) confirmed that its phenotypic characteristics closely paralleled those of a $f l b A^{-}$single mutant and produced an autolytic colony, indicating that $f l b A$ is epistatic to fluG (not shown) for colony development. However, the double mutant sporulation phenotype is more complicated. Both $\mathrm{flbA}^{-}$and $f l u G^{-}$single mutants can respond to $0.8 \mathrm{M} \mathrm{NaCl}$ by producing conidiophores but flbA $A^{-}$flu $G^{-}$double mutants do not (not shown). In contrast, flu $G^{-}$, but not $f l b A^{-}$, mutants conidiate in response to growth-limiting conditions, and the double mutants did make a few conidiophores if growth limitation and high osmolarity were imposed simultaneously.

\section{Discussion}

A. nidulans colony formation is a carefully regulated process involving the organized association of vegetative hyphae within the mycelium and the regulated production of multicellular conidiophores that make thousands of asexual spores (Timberlake 1990; Champe and Simon 1992). For wild-type $A$. nidulans, conidiophore development initiates in the center of a colony within $\sim 24 \mathrm{hr}$ of spore germination, then spreads rapidly toward the edge of the colony so that young conidiophores appear within 1-2 mm of the colony margin, and mature conidiophores are found toward the colony center. Mutations in several distinct genetic loci disrupt programmed initiation of the conidiation pathway, resulting in a fluffy phenotype characterized by the proliferation of undifferentiated aerial hyphae, which give the colony a cotton-like appearance (Roper 1958; Dorn 1970; Yager et al. 1982, Tamame et al. 1983; Adams et al. 1992). The results presented in this paper demonstrate that the fluffy phenotype resulting from deletion of the $f l u G(a c o D)$ gene is rescued and normal conidiophores are produced when flu $G$ deletion mutants are grown next to wild-type conidiating $A$. nidulans strains. In contrast, fluG deletion mutants do not produce conidiophores when grown next to other flu $G^{-}$mutant colonies.

We think that the ability of wild-type strains to rescue the flu $G^{-}$conidiation defect is unlikely to be through genetic exchange because complementation of the mutant phenotype was observed even when the two colonies were separated by a dialysis membrane with $6-8 \mathrm{kD}$ cutoff. In addition, conidia recovered from the rescue zone maintained the flu $G^{-}$mutant phenotype when grown separately. From these results, we propose that flu $G$ is required for production of an extracellular molecule that signals initiation of asexual sporulation by $A$. nidulans. Although this asexual sporulation-inducing factor is likely to be a small, diffusible molecule, our initial attempts to rescue the flu $G^{-}$mutant phenotype by adding extracts prepared from wild-type cultures to growing flu $G^{-}$colonies have failed. Our inability to isolate the sporulation signal in this way might be attributable to low abundance, the presence of antagonists, instability, or an inadequate assay.

Extracellular signals are used by a number of microorganisms for controlling aspects of development and 
other cellular processes (Kim and Kaiser 1990; Willey et al. 1991; Jain et al. 1992). Many fungal species produce specific molecules that function as pheromones in regulating mating pathways, which lead to sexual sporulation, but no such extracellular signals have been clearly implicated in regulating asexual reproduction. Some of these sexual pheromones have been purified and further characterized and include both terpenoids (trisporic acids in the Mucorales, antheridiol in Achlya, and sirenin in the Allomyces) and peptides ( $\alpha$ - and a-factor of $S$. cerevisiae) (Gooday 1983). In addition, $A$. nidulans secretes three distinct unsaturated, hydroxylated, $\mathrm{C} 18$ fatty acid derivatives of linoleic acid called psiA, psiB, and psiC (precocious sexual inducers) that regulate the sexual sporulation pathway. $\mathrm{psiB}$ and $\mathrm{psiC}$ function as positive effectors of sexual sporulation while inhibiting conidiation, but psiA antagonizes $\mathrm{psiB}$ and psiC activity (Champe et al. 1987; Champe and el Zayat 1989; Mazur et al. 1990). These observations have led to the proposal that psiA activates asexual sporulation and that the balance between asexual and sexual reproduction is determined by the relative abundance of $\mathrm{psiA}$ in comparison with psiB and psiC (Champe and el Zayat 1989; Champe and Simon 1992). fluG factor is probably not the same as psiA because addition of purified psiA to flu $G^{-}$mutant strains failed to induce conidiophore development (data not shown). Thus, the asexual sporulation factor produced through fluG activity likely represents a novel signal controlling Aspergillus development.

fluG is predicted to encode an 864-amino-acid polypeptide, and antisera prepared against bacterially produced FluG identified a polypeptide with the predicted $\sim 96-\mathrm{kD}$ molecular mass. The carboxy-terminal half of FluG ( 436 amino acids) has significant similarity to uniquely prokaryotic glutamine synthetase (GSI), whereas the amino-terminal half of the protein is not related to any known sequences in the various data bases (Fig. 3). The similarity observed between the carboxyl terminus of FluG and GSI has important implications regarding both the potential evolutionary relationship of fluG to GSI and the possible mechanisms of FluG action. Although a diverse group of prokaryotes have both GSIand eukaryote-like GSII-encoding genes, fluG represents the first eukaryotic gene that is more closely related to GSI than GSII. Sequence comparisons between the two GS gene types have been used to suggest that GSI and GSII resulted from a gene duplication event that preceded the divergence of eukaryotes and prokaryotes $(\mathrm{Ku}-$ mada et al. 1993). The similarity of FluG to GSI suggests that fluG may too have arisen before the divergence of prokaryotes and eukaryotes. We have detected sequences related to fluG in other closely related fungi, including Aspergillus fumigatus and $A$. niger, but no hybridization was observed to genomic DNA from more distant relatives like Neurospora crassa. It will be interesting to see whether functionally related genes can be isolated from these other organisms.

Despite the observation that the FluG carboxyl terminus resembles glutamine synthetase, the primary function of FluG is clearly not in production of glutamine. flu $G^{-}$mutants are not glutamine auxotrophs, and a second locus $(\operatorname{gn} A)$ has been shown to encode a typical eukaryotic glutamine synthetase (MacDonald 1982; Cornwell and MacDonald 1984). In addition, fluG ${ }^{-}$ $g \ln A^{-}$double mutants have the same glutamine requirement as $g \ln A^{-}$mutants, and addition of glutamine to the media does not suppress the fluffy phenotype of a flu $G^{-}$mutant. Three classically defined $f l u G^{-}$alleles have been shown to map within the carboxyl half of the flu $G$ coding region, and sequence analysis of one of these mutations showed that it resulted from a single amino acid change within the glutamine synthetase domain, supporting the idea that this region is required for normal fluG function (Fig. 3; C. Zimmermann, T. Adams, and L. Yager, unpubl.). One possibility is that FluG has a novel enzymatic function in production of the extracellular sporulation signal and that this function is in some way related to the reaction catalyzed by glutamine synthetase. Given the central role played by glutamine and glutamine synthetase in nitrogen metabolism, it is interesting to speculate that the sporulation signal interferes in some way with nitrogen metabolism, leading to self-regulated nutritional limitation and controlled activation of development. Because FluG is present throughout the life cycle but development is not initiated until at least $24 \mathrm{hr}$ of growth has occurred, either FluG activity is regulated post-translationally or expression of other elements required for response to the developmental signal is controlled. Alternatively, low-level constitutive production of the sporulation signal might provide a means of sensing cell density, with developmental induction only occurring once a threshold level of FluG factor accumulates.

If FluG does not function directly in making the sporulation signal, it could act as a regulator, controlling production of the sporulation signal. In this scenario, the glutamine synthetase domain may act as an effector binding site, and FluG activity might be controlled by sensing cellular levels of glutamine or glutamate. If this were the case, we would expect to find fluffy mutants with defects in genes other than fluG that could be phenotypically rescued in a similar manner. However, none of the other fluffy mutants isolated to date sporulate when grown next to wild-type strains. These mutants all apparently produce the sporulation factor normally as they can rescue the flu $G^{-}$sporulation defect (Fig. 1).

One likely role for the other genes identified by fluffy mutations is to encode products required for responding to the developmental signal made by FluG. In addition to fluG, we have started to characterize other genes identified by fluffy mutations, and a simple model summarizing the potential interactions between these genes in controlling activation of $b r l A$, the central transcriptional regulator of the conidiation pathway, is presented in Figure 6. We propose that fluG is required for production of a signal that initiates a genetically programmed developmental pathway. Because overexpression of $f l b A$ in hyphae results in brlA activation and development, we propose that $f l b A$ functions directly in a regulatory pathway for development (B. Lee and T. Adams, in prep.). The 
Figure 6. Model for fluG in regulating development. We propose that fluG is responsible for production of a signal that functions extracellularly to initiate a pathway leading to activation of $b r l A$ and conidiation. Five other genes $(f l b A, f l b B$, $f l b C, f l b D$, and $f l b E)$ required for response to this signal have been identified by isolation of fluffy mutants, but the nature of their requirement is not clear. In addition, we propose that $f l b A$ has a role in controlling colony development that is distinct from its role in controlling conidiation. flbA is related to $S$. cerevisiae SST2, a gene required for normal regulation of the signaling pathway for response to mating pheromone, and $f 1 b D$ is predicted to encode a DNA-binding protein. All of these fluffy mutants can be phenotypically suppressed suggesting that brlA regulation is complex, involving multiple inputs.

predicted $f l b A$ product is related to the $S$. cerevisiae SST2 product, which in some way regulates the activity of the $G$ protein-mediated signal transduction pathway that controls yeast mating. We propose that FlbA could function like SST2 in controlling the conidiation pathway initiated in response to FluG signal (Dietzel and Kurjan 1987; Kurian 1992). In keeping with this hypothesis, flbA deletion mutants have wild-type levels of FluG polypeptide and are able to rescue the conidiation defect observed in fluG mutants. However, the phenotype of flbA $A^{-}$fluG $G^{-}$double mutants is more complicated than expected for a simple linear pathway initiated by production of the sporulation signal. The hyphal growth phenotype of a $f l b A^{-}$flu $G^{-}$double mutant most closely resembled the $f l b A^{-}$single mutant, suggesting that $f l b A$ functions prior to fluG. These data could be explained if flbA has a role in regulating some aspect of normal hyphal growth that is distinct from its function in responding to the fluG-directed signal that leads to programmed activation of brlA expression. It is also possible that $f l b A$ and flu $G$ are required for activation of separate signaling pathways that have a cumulative effect leading to activation of brlA. These interactions will be made clearer with a better understanding of the genetic requirements for developmental activation resulting from forced overexpression of $f l b A$ (B. Lee and T. Adams, in prep.).

Other fluffy genes are also likely to act in the signal transduction pathway that leads to brlA expression. At least one of these genes, $f l b D$, is predicted to encode a DNA-binding protein, raising the possibility that it acts directly on brlA expression (J. Wieser and T.H. Adams, unpubl.). Finally, there are a number of environmental conditions that can induce $A$. nidulans brlA expression and conidiophore development even in the absence of fluG or other fluffy genes (Adams et al. 1992). The mechanisms connecting these environmental stimuli to the conidiation pathway are complicated and remain to be understood.

\section{Materials and methods}

Fungal strains and growth conditions

All A. nidulans strains used in this study are listed in Table 1.
TTA127.4 and TEP11 were made by transformation of FGSC237 with pTA127 (Adams et al. 1992) and pEP11, respectively. rBN051 was isolated as a meiotic progeny from a cross between TTA127.2 and FGSC33. TAacoll was isolated as a meiotic progeny from a cross between rBN051 and TEP11. RBN127 and RBN130 are progeny of a sexual cross between TBN39.5 and TTA127.2 (Adams et al. 1992). RBN137 was isolated as a meiotic progeny from a cross between HA2 and TTA127.4. Supplemented minimal and complete media were prepared as described (Käfer 1977; Adams et al. 1992).

\section{Genetic techniques}

Standard A. nidulans genetic (Pontecorvo et al. 1953; Clutterbuck 1974) and transformation procedures (Pontecorvo et al. 1953; Clutterbuck 1974; Yelton 1984; Miller et al. 1985) were used.

\section{Nucleic acid isolation and manipulation}

Total RNA was isolated as described previously (Adams et al. 1988 ), separated (10 $\mu \mathrm{g} /$ lane) by electrophoresis on formaldehyde-agarose gels, and transferred without pretreatment to nylon membrane. fluG message was detected by use of a ${ }^{32} \mathrm{P}-\mathrm{la}$ beled random-primed probe generated with an internal $2.5-\mathrm{kb}$ $X$ hol fragment as template. Several fluG cDNA clones were isolated by use of the same fluG-specific probe to screen plaques from a $\lambda$ UNIZAP cDNA library made from RNA from cells grown in log phase (kindly provided by Dr. Greg May, Baylor College of Medicine, Houston, TX). The sequence of the inserts in these clones and of genomic clones was determined by use of standard techniques. To determine the sequence of the fluG684 mutation in TU35, genomic DNA was isolated from the mutant strain and fluG sequences were amplified with the oligonucleotides GGTATCCTCTCATGAAG and ACCCATCAACAGGAGAGG as prin rs for the polymerase chain reaction (PCR). The sequence of the amplified DNA was determined by TAQ cycle sequencing with SequiTherm (Epicentre Technology, Madison, WI).

The fluG::IacZ translational fusion was generated first by insertion of a 3.0-kb BamHI-KpnI lacZ fragment from pRG20 (Han et al. 1993) into BglII and KpnI sites present in pFM1 (Adams et al. 1992) to give pEP9. pEP9 was then digested with KpnI, and a 1.8-kb KpnI fragment from pSH9 (Han et al. 1993) that contains the $5^{\prime}$ end of $\operatorname{trp} C$ was inserted to give pEP11. The fluG::His fusion plasmid (pBN45) for expression and purifica- 
Table 1. A. nidulans strains used in this study

\begin{tabular}{|c|c|c|}
\hline Strain & Genotype & Source \\
\hline FGSC26 & biA1; veA1 & FGSC $^{\mathrm{a}}$ \\
\hline RBN127 & $b i A 1, f l b A:: \arg B ; v e A 1$ & B. Lee and T. Adams (in prep.) \\
\hline TTA127.2 & pabaA1; fluG::trpC; veA1 & Adams et al. (1992) \\
\hline TTA127.4 & pabaA1; fluG::trpC; veA1 & this study \\
\hline TTA125.1 & biA1; fluG::argB; methG1; veA1 & Adams et al. (1992) \\
\hline TEP11 & pabaA1, yA2; trpC::fluG::lacZ, veA1 & this study \\
\hline TAacol1 & pabaA1, yA2; $\Delta$ fluG::trpC; veA1, trpC::fluG::lacZ & this study \\
\hline FGSC237 & $p a b a A 1, y A 2 ; \operatorname{trp} C 801, v e A 1$ & FGSC $^{a}$ \\
\hline TU35 & pabaA1, yA2; fluG684; pyrG89, pyroA4 veA & Adams et al. (1992) \\
\hline rBN051 & biA1; $\triangle$ fluG::trpC; veA1 & this study \\
\hline TA046 & biA1; pyroA4; veA1, $\triangle$ brlA & Adams and Timberlake (1990) \\
\hline TTAl1 & pabaA1, yA2; veA1 & Han et al. $(1993)$ \\
\hline RBN130 & biA1, flbA::arg $B ;$ fluG::trpC; veA1 & this study \\
\hline TBN39.5 & biA1, flbA::argB; methG1; veA1 & B. Lee and T. Adams (in prep.) \\
\hline $\mathrm{HA} 2$ & $y A 2 ; g \ln A 1$, riboE6; veA1 & MacDonald (1982) \\
\hline RBN 137 & pabaA1, yA2; glnA1; fluG::trpC; veA1 & this study \\
\hline FGSC33 & biA1; pyroA4; veA1 & FGSC $^{a}$ \\
\hline
\end{tabular}

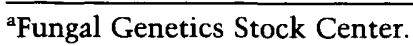

tion of FluG from $E$. coli was constructed first by amplification of a $2.6-\mathrm{kb}$ region corresponding to the FluG-coding sequence by use of a full-length cDNA clone as a template and the oligonucleotides GGTTCATCGTCATGACCAC and GATTAGAGCTCAATACCTC as primers for PCR. The resultant fragment was digested with $B s p H I$ and SstI and cloned into NcoI and SstI sites of pTRC-6H, a derivative of pTRC99A (Pharmacia), allowing the amino-terminal fusion to six histidines in an expression construct (kindly provided by Dr. Bill Dougherty, Oregon State University, Corvallis).

\section{Preparation of protein extracts}

Subcellular fractionation was accomplished by differential centrifugation of total cell lysates. Mycelial cultures of FGSC26 (wild type), TTA127.4 ( $\Delta$ fluG), and RBN127 ( $\Delta f l b A)$ were prepared by inoculation of $200 \mathrm{ml}$ of complete medium with $1 \times 10^{5}$ spores $/ \mathrm{ml}$ and growth at $37^{\circ} \mathrm{C}$ for $20 \mathrm{hr}$ with constant shaking $(300 \mathrm{rpm})$. Cultures were harvested by filtration, and the resulting hyphal mats were broken up and placed in a beaker on ice. The mycelial pieces were mixed with SSE0.5 buffer $140 \mathrm{~mm}$ spermidine- $\mathrm{HCl}, 1 \mathrm{mM}$ spermine- $\mathrm{HCl}, 100 \mathrm{mM} \mathrm{KCl}, 10 \mathrm{~mm}$ EDTA, $10 \mathrm{~mm}$ Tris- $\mathrm{HCl}$ at $\mathrm{pH}$ 7) to form a thick mycelial paste. The mycelial paste was ground under liquid nitrogen with a mortar and pestle to produce a fine powder. The frozen mycelial powder was quickly thawed and transferred to a precooled Dounce homogenizer. Following homogenization, cell debris was removed by filtration through two layers of Miracloth and by centrifugation of the filtrate at least three times at $1000 \mathrm{~g}$ for $10 \mathrm{~min}$. The nuclear pellet was isolated by centrifugation at $6000 \mathrm{~g}$ for $20 \mathrm{~min}$, and the pellet was washed three times in SSE0.5. The mitochondrial pellet was isolated from the postnuclear fraction by centrifugation for $30 \mathrm{~min}$ at $20,000 \mathrm{~g}$ followed by three washes in SSE0.5. A crude membrane fraction was prepared from the postmitochondrial supernatant by centrifugation for $1 \mathrm{hr}$ at $100,000 \mathrm{~g}$ followed by three washes in SSE0.5. The supernatant from this step was saved as the soluble cytoplasmic fraction. Each pellet was suspended in sonication buffer (25\% glycerol, $200 \mathrm{mM} \mathrm{NaH} \mathrm{PO}_{4}$ at $\mathrm{pH} 7,1 \mathrm{mM} \mathrm{PMSF}$ ), and organelles were lysed by sonication $(2 \times 15$-sec bursts). Protein concentration was determined as described by Bradford (1976).
The presence of DNA in each fraction was determined by measuring fluorescence of Hoechst 33258 to show that $>95 \%$ of the total DNA in the sample was recovered in the nuclear and mitochondrial pellets.

For preparation of total cell extracts, mycelial cultures were grown as described above, and development was induced following $20 \mathrm{hr}$ of growth in liquid culture as described previously (Adams et al. 1988). Samples were collected $8 \mathrm{hr}$ before inducing development ( $12 \mathrm{hr})$, the time development was induced $(20 \mathrm{hr})$, and $8 \mathrm{hr}$ after inducing development $(28 \mathrm{hr})$ then frozen in liquid nitrogen and lyophilized. Samples were ground to a fine powder and suspended in lysis buffer $\left(200 \mathrm{mM} \mathrm{NaH}_{2} \mathrm{PO}_{4}\right.$ at pH 7, 25\% glycerol, $1 \mathrm{mM}$ PMSF, $0.1 \%$ SDS), and the supernatant was collected following centrifugation. Protein concentration was determined as described by Lowry (Lowry et al. 1951).

\section{Isolation of FluG fusion protein and antisera}

E. coli cells containing the FluG::6His plasmid, pBN45, were grown in $500 \mathrm{ml}$ Luria broth medium at $37^{\circ} \mathrm{C}$ with vigorous shaking to an $\mathrm{OD}_{600}$ of 0.6 , and expression was induced by addition of IPTG to a final concentration of $0.3 \mathrm{~mm}$. Cells were incubated for $2 \mathrm{hr}$ and then pelleted by centrifugation at $6000 \mathrm{~g}$ for $5 \mathrm{~min}$. The pellet was washed with $50 \mathrm{ml}$ of PBS and then resuspended in $5 \mathrm{ml}$ of $100 \mathrm{~mm}$ Tris- $\mathrm{HCl}(\mathrm{pH} 7.5)$. The suspension was frozen at $-20^{\circ} \mathrm{C}$ for $1 \mathrm{hr}$, and cells were lysed as a result of being allowed to thaw at room temperature. Because the majority of the FluG::6His fusion protein was present as an insoluble pellet, we suspended this pellet in denaturation buffer (6 $\mathrm{M}$ Guanidine- $\mathrm{HCl}, 0.1 \mathrm{M} \mathrm{NaH}_{2} \mathrm{PO}_{4}, 10 \mathrm{~mm}$ Tris- $\mathrm{HCl}$ at $\mathrm{pH} 8$ ). The denatured FluG::6His fusion protein was purified by binding to Ni-NTA followed by elution with $250 \mathrm{~mm}$ immidazole (Hochuli et al. 1988; Janknecht et al. 1991). Approximately $7 \mathrm{mg}$ of purified FluG protein was obtained from the $500-\mathrm{ml}$ culture. For preparation of polyclonal antibodies from rabbits, $250 \mu \mathrm{g}$ of pure FluG::His fusion protein was used in the initial immunization and in each subsequent treatment.

\section{Immunoblot analysis}

Polyacrylamide gel $(8 \%)$ electrophoresis was carried out as described by Laemmli (1970). Proteins (50 $\mu \mathrm{g} /$ lane) were electro- 
phoretically transferred to Immobilon-P membrane (Millipore) in $20 \mathrm{~mm}$ Tris- $\mathrm{HCl}, 150 \mathrm{~mm}$ glycine, and $20 \%$ methanol by use of a semidry blotter at $0.8 \mathrm{~mA} / \mathrm{cm}^{2}$. The blots were blocked by incubation in $5 \%$ nonfat dry milk in TTBS $(20 \mathrm{~mm}$ Tris- $\mathrm{HCl}$ at $\mathrm{pH} 7.5,150 \mathrm{~mm} \mathrm{NaCl}, 0.1 \%$ Tween 20 ) for at least $\mathrm{l}$ hr at room temperature followed by a 2 -hr incubation at room temperature with FluG-specific antisera diluted 1:20,000 in TTBS with 5\% nonfat dry milk. Blots were washed at least three times with TTBS and then incubated for $1 \mathrm{hr}$ at room temperature with a 1:8000 dilution of goat anti-rabbit secondary antibody conjugated with horseradish peroxidase. After three washes in TTBS, horseradish peroxidase activity was detected by use of an Enhanced Chemiluminescence detection kit (Amersham International, England).

\section{Acknowledgments}

This work was supported by National Institutes of Health grant GM45252-01 and Texas Advanced Research Program grant 010366-79 to T.H.A.. We thank Drs. Debbie Siegele, Dan Ebbole, and Rodolfo Aramayo for critically evaluating the manuscript, Dr. Sewell Champe for providing purified psiA factor, Ed Pestovic for his technical assistance, and Dr. Mike Plamann and our colleagues in the laboratory for many helpful discussions.

The publication costs of this article were defrayed in part by payment of page charges. This article must therefore be hereby marked "advertisement" in accordance with 18 USC section 1734 solely to indicate this fact.

\section{References}

Adams, T.H., M.T. Boylan, and W.E. Timberlake. 1988. brlA is necessary and sufficient to direct conidiophore development in Aspergillus nidulans. Cell 54: 353-362.

Adams, T.H., W.A. Hide, L.N. Yager, and B.N. Lee. 1992. Isolation of a gene required for programmed initiation of development by Aspergillus nidulans. Mol. Cell. Biol. 12: 38273833.

Almassy, R.J., C.A. Janson, R. Hamlin, N.-H. Xuong, and D. Eisenberg. 1986. Novel subunit-subunit interactions in the structure of glutamine synthetase. Nature 323: 304-309.

Altschul, S.F., W. Gish, W. Miller, W.W. Myers, and D.J. Lipman. 1990. Basic local alignment search tool. J. Mol. Biol. 215: 403-410.

Axelrod, D.E. 1972. Kinetics of differentiation of conidiophores and conidia by colonies of Aspergillus nidulans. I. Gen. Microbiol. 73: 181-184.

Boylan, M.T., P.M. Mirabito, C.E. Willett, C.R. Zimmerman, and W.E. Timberlake. 1987. Isolation and physical characterization of three essential conidiation genes from Aspergillus nidulans. Mol. Cell. Biol. 7: 3113-3118.

Bradford, M.M. 1976. A rapid and sensitive method for the quantitation of microgram quantities of protein utilizing the principle of protein-dye binding. Anal. Biochem. 72: 248254.

Champe, S.P. and A. el Zayat. 1989. Isolation of a sexual sporulation hormone from Aspergillus nidulans. J. Bacteriol. 171: 3982-3988.

Champe, S.P., and L.D. Simon. 1992. Cellular differentiation and tissue formation in the fungus Aspergillus nidulans. In Morphogenesis: An analysis of the development of biological form (ed. E.F. Rossomando and S. Alexander), pp. 63-91. Marcel Dekker, New York.

Champe, S.P., P. Rao, and A. Chang. 1987. An endogenous in- ducer of sexual development in Aspergillus nidulans. J. Gen. Microbiol. 133: 1383-1387.

Clutterbuck, A.J. 1969. A mutational analysis of conidial development in Aspergillus nidulans. Genetics 63: 317-327.

- 1974. Aspergillus nidulans. In Handbook of Genetics (ed. R.C. King), pp. 447-510.. Plenum Press, New York

Cornwell, E.V. and D.W. MacDonald. 1984. glnA mutations define the structural gene for glutamine synthetase in $A s$ pergillus. Curr. Genet. 8: 33-36.

Dietzel, C. and J. Kurjan. 1987. Pheromonal regulation and sequence of the Saccharomyces cerevisiae SST2 gene: A model for desensitization to pheromone. Mol. Cell. Biol. 7: 41694177.

Dorn, G.L. 1970. Genetic and morphological properties of undifferentiated and invasive variants of Aspergillus nidulans. Genetics 66: 267-279.

Gooday, G.W. 1983. In Secondary metabolism and differentiation in fungi (ed. J.W. Bennett and A. Ciegler), pp. 239-266. Marcel Dekker, New York.

Han, S., J. Navarro, R.A. Greve, and T.H. Adams. 1993. Translational repression of $b r l A$ expression prevents premature development in Aspergillus. EMBO J. 12: 2449-2457.

Hochuli, E., W. Bannwarth, H. Dobeli, R. Gentz, and D. Stuber. 1988. Genetic approach to facilitate purification of recombinant proteins with a novel metal chelate. BioTechnology 6: $1321-1325$.

Jain, R., I.S. Yuen, C.R. Taphouse, and R.H. Gomer. 1992. A density-sensing factor controls development in Dictyostelium. Genes \& Dev. 6: 390-400.

Janknecht, R., G. De Marynoff, J. Lou, R.A. Hipskind, A. Nordheim, and H.G. Stunnenberg. 1991. Rapid and efficient purification of native histidine-tagged protein expressed by recombinant vaccinia virus. Proc. Natl. Acad. Sci. 88: 89728976.

Janssen, P.J., W.A. Jones, D.T. Jones, and D.R. Woods. 1988. Molecular analysis and regulation of the $\operatorname{gn} A$ gene of the gram-positive anaerobe Clostridiumm acetabutylicum. I. Bacteriol. 170: 400-408.

Käfer, E. 1977. Meiotic and mitotic recombination in Aspergillus and its chromosomal aberrations. Adv. Genet, 19:33131.

Kim, S.K. and D. Kaiser. 1990. C-factor: A cell signaling protein required for fruiting body morphogenesis of $M$. xanthus. Cell 61: 19-26.

Kumada, Y., D.R. Benson, D. Hillemann, T.J. Hosted, D.A. Rochefort, D.J. Thompson, W. Wohlleben, and Y. Tateno. 1993. Evolution of the glutamine synthetase gene, one of the oldest existing and functioning genes. Proc. Natl. Acad. Sci. 90: 3009-3013.

Kurjan, J. 1992. Pheromone response in yeast. Annu. Rev. Biochem. 61: 1097-1129.

Laemmli, U.K. 1970. Cleavage of structural proteins during the assembly of the head of bacteriophage T4. Nature 227: 680685.

Lowry, O.H., N.J. Rosebrough, A.L. Farr, and R.J. Randall. 1951. Protein measurement with the Folin phenol reagent. J. Biol. Chem. 193: 265-275.

MacDonald, D.W. 1982. A single mutation leads to loss of glutamine synthetase and relief of ammonium repression in Aspergillus. Curr. Genet. 6: 203-208.

Mazur, P., H.V. Meyers, K. Nakanishi, A.E. El-Zayat, and S.P. Champe. 1990. Structural elucidation of sporogenic fatty acid metabolites in Aspergillus nidulans. Tetrahedron Lett. 31: 3837 .

Miller, B., K. Miller, and W. Timberlake. 1985. Direct and indirect gene replacements in Aspergillus nidulans. Mol. Cell. 
Biol. 5: 1714-1721.

Nakano, Y., C. Kato, E. Tanaka, K. Kimura, and K. Hoikoshi. 1989. Nucleotide sequence of the glutamine synthetase gene $(g \ln A)$ and its upstream region from Bacillus cereus. I. Biochem. 106: 209-215.

Pontecorvo, G., J.A. Roper, L.M. Hemmons, K.D. MacDonald, and A.W.J. Bufton. 1953. The genetics of Aspergillus nidulans. Adv. Genet. 5: 141-238.

Possot, O., L. Sibold, and J.P. Aubert. 1989. Nucleotide sequence and expression of the glutamine synthetase gene, $g \ln A$, of the archaebacterium Methanococcus voltae. Res. Microbiol. 140: 355-371.

Roper, J.A. 1958. Nucleo-cytoplasmic interactions in Aspergillus nidulans. Cold Spring Harbor Symp. Quant. Biol. 23: $141-154$.

Tamame, M., F. Antequera, J.R. Villanueva, and T. Santos. 1983. High-frequency conversion of a "fluffy" developmental phenotype in Aspergillus spp. by 5-azacytidine treatment: Evidence for involvement of a single nuclear gene. Mol. Cell. Biol. 3: 2287-2297.

Timberlake, W.E. 1990. Molecular genetics of Aspergillus development. Annu. Rev. Genet. 24: 5-36.

Willey, J., R. Santamaria, J. Guijarro, M. Geistlich, and R. Losick. 1991. Extracellular compementation of a developmental mutation implicates a small sporulation protein in aerial mycelium formation by $S$. coelicolor. Cell 65: 641650.

Yager, L.N., M.B. Kurtz, and S.P. Champe. 1982. Temperatureshift analysis of conidial development in Aspergillus nidulans. Dev. Biol. 93: 92-103.

Yelton, M.M., J.E. Hamer, and W.E. Timberlake. 1984. Transformation of Aspergillus nidulans by using a $\operatorname{trp} C$ plasmid. Proc. Natl. Acad. Sci. 81: 1470-1474. 


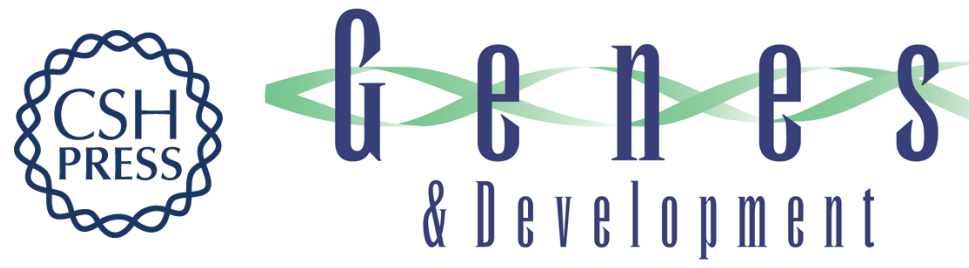

\section{The Aspergillus nidulans fluG gene is required for production of an extracellular developmental signal and is related to prokaryotic glutamine synthetase $\mathrm{I}$.}

B N Lee and T H Adams

Genes Dev. 1994, 8:

Access the most recent version at doi:10.1101/gad.8.6.641

References This article cites 36 articles, 15 of which can be accessed free at: http://genesdev.cshlp.org/content/8/6/641.full.html\#ref-list-1

License

Email Alerting Service

Receive free email alerts when new articles cite this article - sign up in the box at the top right corner of the article or click here.

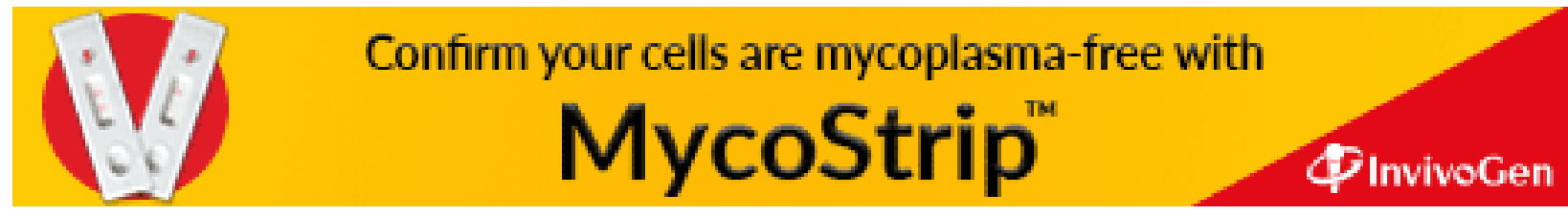

